\title{
Unsupervised correction of gene- independent cell responses to CRISPR-Cas9 targeting
}

\author{
Francesco lorio ${ }^{1,2,5^{*}{ }^{\dagger}}$, Fiona M. Behan ${ }^{2,5 \dagger}$, Emanuel Gonçalves ${ }^{2}$, Shriram G. Bhosle ${ }^{2}$, Elisabeth Chen ${ }^{2}$, \\ Rebecca Shepherd ${ }^{2}$, Charlotte Beaver ${ }^{2}$, Rizwan Ansari ${ }^{2}$, Rachel Pooley², Piers Wilkinson², Sarah Harper², \\ Adam P. Butler ${ }^{2}$, Euan A. Stronach ${ }^{3,5}$, Julio Saez-Rodriguez ${ }^{1,4,5,6+}$, Kosuke Yusa ${ }^{2+}$ and Mathew J. Garnett ${ }^{2,5^{*+}}$
}

\begin{abstract}
Background: Genome editing by CRISPR-Cas9 technology allows large-scale screening of gene essentiality in cancer. A confounding factor when interpreting CRISPR-Cas9 screens is the high false-positive rate in detecting essential genes within copy number amplified regions of the genome. We have developed the computational tool CRISPRcleanR which is capable of identifying and correcting gene-independent responses to CRISPR-Cas9 targeting. CRISPRcleanR uses an unsupervised approach based on the segmentation of single-guide RNA fold change values across the genome, without making any assumption about the copy number status of the targeted genes.

Results: Applying our method to existing and newly generated genome-wide essentiality profiles from 15 cancer cell lines, we demonstrate that CRISPRcleanR reduces false positives when calling essential genes, correcting biases within and outside of amplified regions, while maintaining true positive rates. Established cancer dependencies and essentiality signals of amplified cancer driver genes are detectable post-correction. CRISPRcleanR reports sgRNA fold changes and normalised read counts, is therefore compatible with downstream analysis tools, and works with multiple sgRNA libraries.
\end{abstract}

Conclusions: CRISPRcleanR is a versatile open-source tool for the analysis of CRISPR-Cas9 knockout screens to identify essential genes.

Keywords: CRISPR-Cas9, Genetic screens, Cancer, Gene copy number, Bias correction

\section{Background}

CRISPR-Cas9-based genome editing techniques are transforming the landscape of genetic studies $[1,2]$. The high efficiency and specificity of the CRISPR-Cas9 system to mutagenise genes through the introduction of DNA double strand breaks (DSB), either at the level of individual genes or at genome-wide scale, enables the systematic investigation of loss-of-function phenotypes.

We and others have developed genome-wide pooled CRISPR knock-out (CRISPR-KO) screening strategies

\footnotetext{
* Correspondence: fi1@sanger.ac.uk; mg12@sanger.ac.uk

†Francesco lorio, Fiona M. Behan, Julio Saez-Rodriguez, Kosuke Yusa and Mathew J. Garnett contributed equally to this work.

${ }^{1}$ European Molecular Biology Laboratory - European Bioinformatics Institute, Cambridge, UK

${ }^{2}$ Wellcome Sanger Institute, Cambridge, UK

Full list of author information is available at the end of the article
}

[3-5]. A prominent application of CRISPR-KO screens is the systematic identification of genes that are essential for cancer cell fitness to identify strategies for the development of novel targeted therapies. These studies typically introduce Cas9 endonuclease into cells, followed by or alongside the introduction of a library of pooled sgRNAs targeting the genome. The library usually contains multiple single guide RNA (sgRNA) targeting each gene to facilitate a robust identification of essential genes. Analysis strategies compare the abundance of sgRNAs between control and test samples to determine which sgRNAs are differentially represented, thus targeting a gene that is potentially essential to the fitness of the cancer cells. Several groups have performed these types of screens to identify novel drug targets $[6,7]$. A recent landmark study has reported gene essentialities in

(c) The Author(s). 2018 Open Access This article is distributed under the terms of the Creative Commons Attribution 4.0 International License (http://creativecommons.org/licenses/by/4.0/), which permits unrestricted use, distribution, and 
342 cancer cell lines [8]. This will empower association studies between gene essentialities and genomic/transcriptomic features to develop biomarkers for patient stratification.

One drawback of the CRISPR-KO screening system is caused by its mode of action, namely DSB induction. DSBs trigger a DNA damage response which can cause cell cycle arrest and in some cases cell death [9-11]. This is problematic when performing whole-genome CRISPR-KO screens in cancer cells because of frequent copy number $(\mathrm{CN})$ alterations in their genome, resulting in widespread Cas9 induced DNA damage. Consequently, DSBs at genes in amplified regions result in depletion of these genes in a pooled CRISPR-KO screen regardless of their essentiality, and thus they are erroneously called as fitness genes. This can result in a high false-positive rate and correcting for this $\mathrm{CN}$-associated effect is crucial for the interpretation of CRISPR-KO screening results. Solutions proposed thus far encompass scanning the dataset for biased regions and their removal from downstream analysis [12], resulting in the exclusion of potentially biologically relevant genes residing in $\mathrm{CN}$-amplified regions, or to apply a piecewise linear model to infer true gene dependencies based on $\mathrm{CN}$ profiles across large panels of cell lines [8].

During the analysis of CRISPR-KO data we identified a number of instances for which existing approaches for correcting bias in CRISPR-KO data were unsuitable or hampered further downstream analyses. To address this, we developed CRISPRcleanR, a computational approach implemented in open-source $\mathrm{R}$ and a Python packages, which identifies biased genomic regions from CRISPR-KO screens in an unsupervised manner and provides both corrected read count and $\log$ fold change $(\log \mathrm{FC})$ values of individual sgRNAs in such regions. Our method reduces false positive calls while keeping the true positive rate of known essential genes largely unchanged, and allows the detection of essential genes even within focally amplified regions.

\section{Results}

\section{Gene-independent responses in CRISPR-KO screens}

We performed genome-wide CRISPR-KO screens on 15 human cancer cell lines (hereafter called 'Project Score'), which are a subset of the Genomics of Drug Sensitivity in Cancer (GDSC) collection (Additional file 1: Table S1) $[13,14]$. This involved six tumour types with different mutational processes, including high frequency of single-nucleotide variants (large intestine, lung, and melanoma) and $\mathrm{CN}$ variation (breast and ovary). We used the Sanger Institute CRISPR library (version 1.0) targeting 18,010 genes (90,709 sgRNAs; $\sim 5$ sgRNAs per gene) [6]. The screens showed high consistency between technical replicates in each cell line (median average correlation for sgRNA counts $=0.83$ ) and readily discriminated between pre-defined fitness essential (FE) and non-essential genes (median area under the Receiver Operating Characteristic curve $($ AUROC) $=0.92)$ (Additional file 2: Figure S1) [15]. Additionally, a high true positive rate (TPR, or recall) was observed for known essential genes assembled from the Molecular Signature Database (MsigDB) [16] and from literature [17] (median TPR across gene sets and cell lines $=85 \%$ at $5 \%$ FDR).

When comparing CRISPR data and $\mathrm{CN}$ profiles for each line, we confirmed a large negative effect for logFCs of sgRNAs targeting genes in $\mathrm{CN}$-amplified regions, particularly with $\mathrm{CN} \geq 8$ (Additional file 2: Figure $\mathrm{S} 2$ and Additional file 1: Table S2). Notably, sgRNA targeting $\mathrm{CN}$-amplified $(\mathrm{CN} \geq 8)$ non-expressed genes (FPKM < $0.05)$ were significantly more depleted in six cell lines than the rest of the sgRNA in the whole library. For three cell lines (HT55, EPLC-272H, and MDA-MB-415), the negative effect on $\operatorname{logFC}$ of sgRNA in $\mathrm{CN}$-amplified regions was comparable or greater than for $\mathrm{FE}$ genes (Additional file 2: Figure S3 and Additional file 1: Table S2). Collectively, using independent data, our analysis confirms the systematic negative bias on sgRNA logFC values in particular regions of the genome, which are enriched for $\mathrm{CN}$ amplifications.

\section{Variable effect of amplification on responses to CRISPR- Cas9 targeting}

To gain greater insight into $\mathrm{CN}$-associated biases, we performed a detailed analysis of the relationship between sgRNA $\log F C$ values and $\mathrm{CN}$ at the level of individual $\mathrm{CN}$ segments (Fig. 1a and Additional file 2: Figure S4). For some cell lines, the negative bias on average logFC values within segments was positively correlated with $\mathrm{CN}$ values (EPLC-272H, NCI-H520, OVCAR-8, TOV-21G and SW48). In other cell lines the bias effect on average logFC was not observed (MDA-MB-436), plateaued (NCI-H2170), or fluctuated as $\mathrm{CN}$ varied (MDA-MB-453, HT55 and HuP-T3). These effects were preserved when only considering sgRNA targeting non-expressed genes (Fig. $1 \mathrm{~b}$ and Additional file 2: Figure S4), demonstrating that the negative $\log \mathrm{FCs}$ are most likely independent of true gene essentiality. In addition, we observed a wide range of average $\log \mathrm{FC}$ values for segments of a given CN (Fig. 1a, b), and this is often larger than the variation between segments of different $\mathrm{CN}$, indicating that $\mathrm{CN}$ alone does not capture all of the observed bias variance.

Furthermore, although in the majority of instances CN segments matched segments of equal sgRNA logFCs (Fig. 1c), we identified several $\mathrm{CN}$ segments with discontinuous $\operatorname{logFC}$ patterns (Fig. 1d). Additionally, regions of consistently depleted sgRNAs were identified also in diploid regions of the genome. For example, the cell line CL-40 harbours two copies of chromosome 16, but several contiguous genes (of which many are not expressed) 


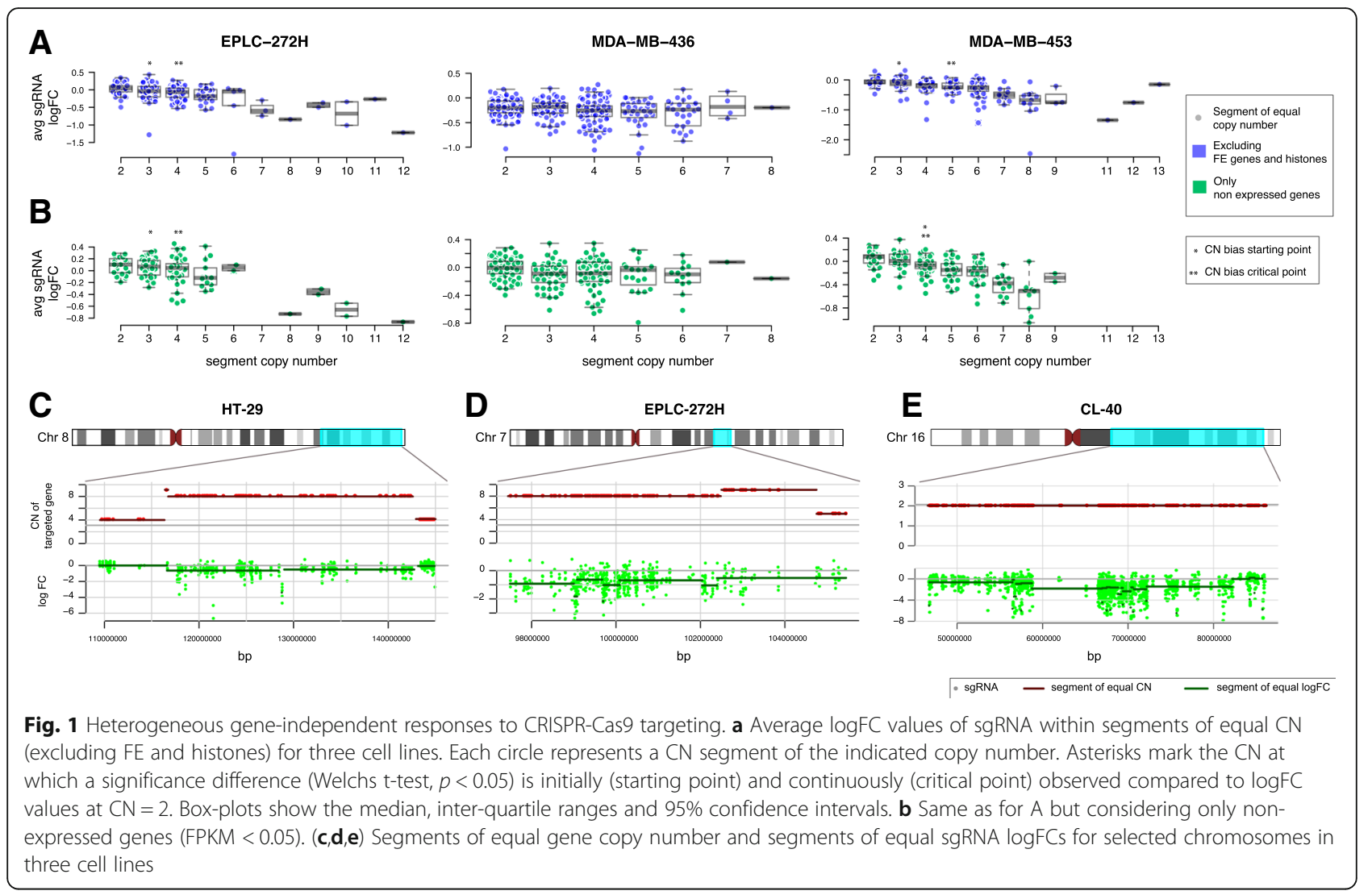

in region 16q23 exhibited a negative $\log \mathrm{FC}$ across targeting guides (Fig. 1e).

Our results indicate that biases observed in CRISPR-KO screens are often associated with $\mathrm{CN}$ alterations but are heterogeneous, with poorly understood variation between segments of differing $\mathrm{CN}$, and variation within segments of the same $\mathrm{CN}$. Taken together, these results highlights the value of an unsupervised approach, not dependent on $\mathrm{CN}$ alone, to correct for biased regions in CRISPR-KO data.

\section{CRISPRcleanR corrects bias in CRISPR-Cas9 datasets}

In order to detect biased regions in an unsupervised manner and correct corresponding sgRNA logFCs in CRISPR-KO screening data, we developed CRISPRcleanR, a computational approach implemented in open-source $\mathrm{R}$ and Python packages. CRISPRcleanR applies a circular binary segmentation algorithm, originally developed for array-based comparative genomic hybridization assay $[18,19]$, directly to the genome-wide patterns of sgRNA logFCs across individual chromosomes in a cell line. It then detects genomic segments containing multiple sgRNAs with sufficiently equal $\operatorname{logFC}$. If these segments contain sgRNAs targeting a minimum number of distinct genes then the sgRNA in the segment are most likely responding to CRISPR-Cas9 targeting in a gene-independent manner, and logFCs values are corrected via mean-Centering. Median-based centering can also be applied for experimentally variable data or in the presence of many outliers.

CRISPRcleanR embeds functions from the DNAcopy $\mathrm{R}$ package [20] allowing users to customise their arguments. Furthermore, it has several features that make it statistically robust, versatile and practical for downstream applications: (i) it works in an unsupervised manner, requiring no chromosomal $\mathrm{CN}$ information nor a priori defined sets of essential genes; (ii) it implements a $\log \mathrm{FC}$ correction, making depletion scores for all genes usable in follow up analyses; (iii) it examines $\log \mathrm{FC}$ at the sgRNA level to gain resolution and to account for different levels of sgRNA on-target efficiency, and enables the subsequent use of algorithms to call gene depletion significance that require input data at the sgRNA level (e.g. BAGEL [21]); (iv) by applying an inverse transformation to corrected sgRNA logFCs, it computes corrected sgRNA counts, which are required as input for commonly used mean-variance modeling approaches, such as MAGeCK [22], to call gene depletion/enrichment significance; (v) lastly, CRISPRcleanR corrects $\operatorname{logFC}$ values using data from an individual cell line and with invariant performances, unlike other computational correction approaches whose performances depend on the number of analysed cell lines [8]; as a consequence, 
CRISPRcleanR is suitable for the analysis of data from both small- and large-scale CRISPR-KO studies.

When applied to Project Score data, CRISPRcleanR effectively corrected the bias in sgRNA logFCs over a wide range of chromosomal segments with variable $\mathrm{CN}$ alterations. Furthermore, this included detection and correction of different level of biases in sgRNA logFCs within an individual segment of equal CN (Fig. 2a, b). An immediate result of the application of CRISPRcleanR to our data was that biases in particularly high $\mathrm{CN}$ regions were strongly attenuated over all the cell lines (Fig. 2c).
Overall, CRISPRcleanR reduced the recall of sgRNAs targeting $\mathrm{CN}$-amplified regions, including sgRNAs targeting $\mathrm{CN}$-amplified non-expressed genes, towards expectation when classifying the whole library of sgRNAs based on their logFCs (Fig. 2d). The correction was also consistently observed at the gene level (average $\operatorname{logFCs}$ of targeting sgRNAs) across all screened cell lines at a fixed 5\% FDR, with a median reduction in recall equal to $72 \%$ and $88 \%$, respectively for $\mathrm{CN}$-amplified and $\mathrm{CN}$-amplified non-expressed genes (Fig. $2 \mathrm{e}$ and Additional file 1: Table S3). This reduction was also

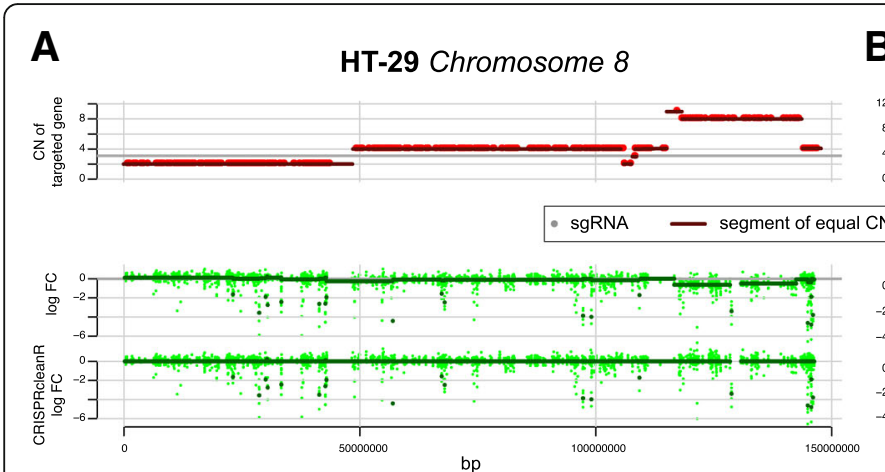

B

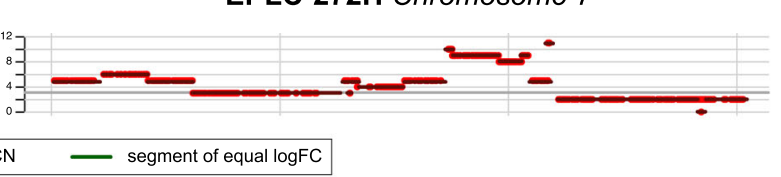

C All cell Lines Pre CRISPRcleanR

All cell Lines Post CRISPRcleanR
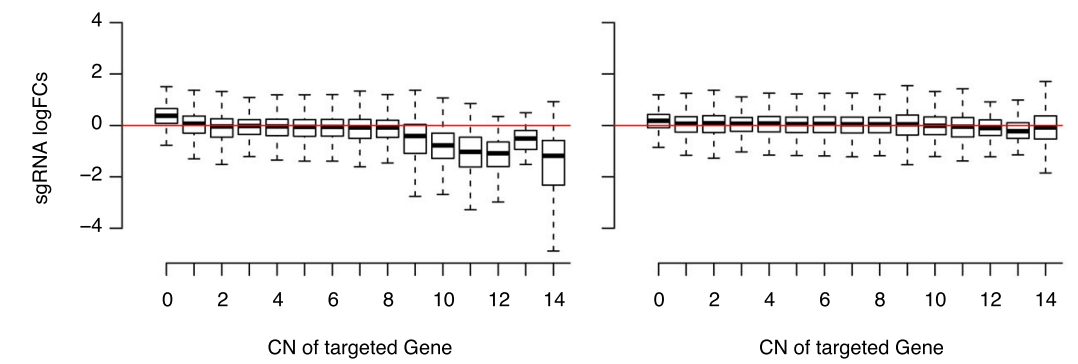

D

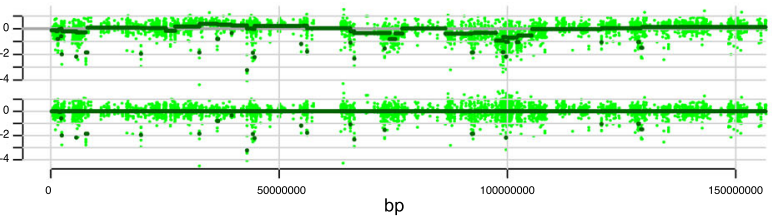

E
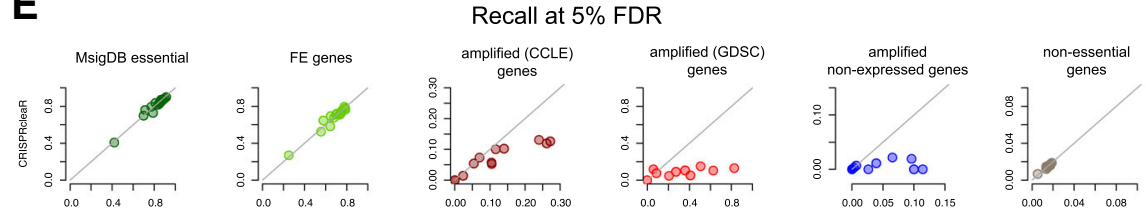

Area under Recall curve
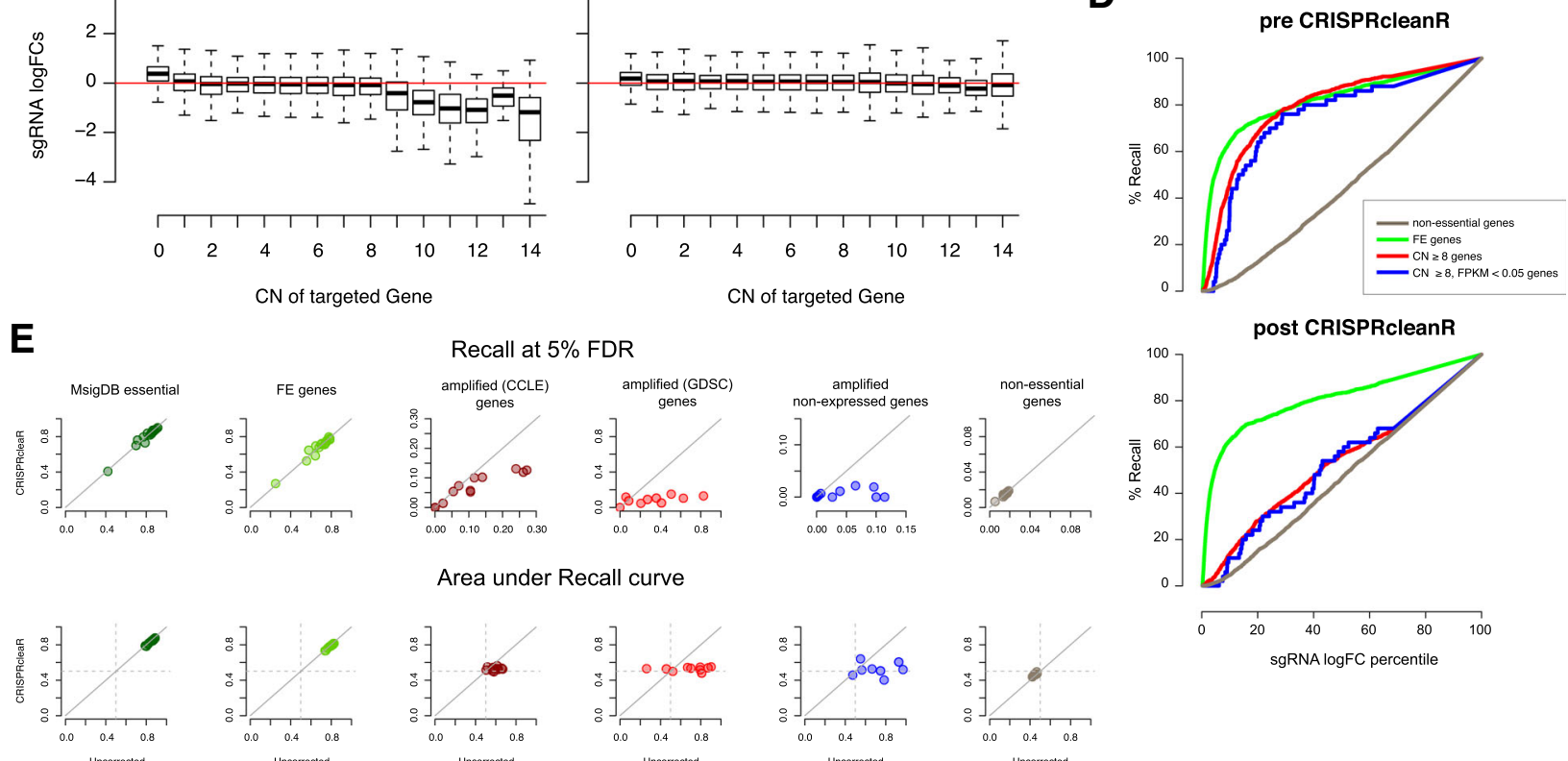

Fig. 2 Unsupervised detection of segments of equal sgRNA logFCs and their correction. $\mathbf{a}$ and $\mathbf{b}$ Example segments of equal gene copy number and equal sgRNA logFC values detected and corrected by CRISPRcleanR in two cell lines. c logFC values of sgRNAs of the entire library for all cell lines grouped according to the copy number of their targeted gene before (left) and after (right) CRISPRcleanR correction. Box-plots show the median, inter-quartile ranges and 95\% confidence intervals. $\mathbf{d}$ Recall curves of sgRNA when classified as targeting amplified genes, amplified non-expressed genes, FE genes, and non-essential genes before and after CRISPRcleanR correction, for an example cell line (EPLC-272H). e Assessment of CRISPRcleanR correction comparing Recall at 5\% FDR (top row) or area under the Recall curve (AURC, bottom row) of genes in six predefined gene sets based on their uncorrected or corrected logFCs (averaged across targeting sgRNAs) 
observed at the level of the area under the overall recall curves (AURCs), thus independent of a fixed depletion significance threshold. Specifically, we observed the median AURCs across all cell lines shifting from 0.74 to 0.51 ( $p=0.02$, Welch's two sample t-test $)$ and from 0.7 to $0.5(p=0.01)$, respectively, for $\mathrm{CN}$-amplified and $\mathrm{CN}$-amplified non-expressed genes (Fig. 2e and Additional file 1: Table S3). The reduction in AURC was independent of whether amplified genes in cell lines were identified using $\mathrm{CN}$ data from the GDSC or the cancer cell line encyclopedia (CCLE). In contrast, for the MsigDB known essential genes and the FE genes, the reduction was negligible at less than $2 \%$, with median AURCs preserved at $\geq 0.82$.

Excluding from the essentiality profiles the sgRNAs targeting a priori known essential genes (taken from MSigDB) before CRISPRcleanR correction yielded very similar results as when imposing the constraint that, for a segment to be corrected, it must contain sgRNA targeting $n=3$ different genes (Additional file 2: Figure S5). This was determined by performing several correction attempts varying $n$ and considering or not FE and other MSigDB essential genes. Thus, CRISPRcleanR can be used in a completely unsupervised setting, without making any assumption on gene essentiality.

\section{CRISPRcleanR is effective using multiple sgRNA libraries}

To investigate the versatility of CRISPRcleanR we assessed its performance across different libraries of sgRNAs. For the purpose of comparability we initially used our previously published dataset derived from screening the HT-29 cell line with the Brunello [23] and Whitehead [12] libraries, using the same lentiviral vector as our library [24]. Of note, despite all three libraries targeting 17,646 overlapping genes, fewer than $5 \%$ of the 19-mer gRNA in the libraries are overlapping in sequence. A similar reductions in recall for $\mathrm{CN}$-amplified genes (mean $=40 \pm 2.7 \%$ ), $\mathrm{CN}$ amplified non-expressed genes (45 $\pm 5.7 \%)$, fitness essential genes $(2 \pm 0.47 \%)$, and non-essential genes (mean $=-3.8 \pm 1.81 \%$ ) was observed across all three libraries (Fig. 3a, b). As a specific example, all three libraries showed matching patterns of biased logFCs in the same $\mathrm{CN}$-amplified genomic region spanning the proto-oncogene $M Y C$ on chromosome 8 (Fig. 3c). CRISPRcleanR corrected the sgRNA $\log F C$ values for this bias in all three libraries.

To further evaluate the compatibility of CRISPRcleanR with different sgRNA libraries, we tested it on an independent dataset of 342 cell lines using the Avana library from Project Achilles [8] (Additional file 2: Figure S6). We observed a reduction of false positive hits (average recall at $5 \%$ FDR) for $\mathrm{CN}$-amplified genes after correction from 0.10 to $0.04\left(p=6.23 \times 10^{-29}\right)$ based on GISTIC [25] copy number scores from the CCLE, from 0.27 to $0.08\left(p=1.64 \times 10^{-8}\right)$ based on PicNic [26] copy number scores from the GDSC [13], and from 0.03 to $0.001\left(p=10^{-4}\right)$ for non-expressed genes which are $\mathrm{CN}$-amplified according to either GISTIC or PicNic scores. Additionally, true positive rates for known essential genes were slightly increased (average recall at 5\% FDR) for a priori known essential genes from MSigDB [16] from 0.74 to $0.76(p=0.06)$, and significantly increased for essential genes from [15] from 0.59 to 0.63 $\left(p=8 \times 10^{-4}\right.$, Additional file 2: Figure S6). The recall increment for known essential genes was greatest for lower quality CRISPR-KO data, suggesting that CRISPRcleanR contributes to a signal improvement in noisy or low quality data (Additional file 2: Figure S7). Taken together, these results show that CRISPRcleanR is suitable for correcting bias in CRISPR-KO screening datasets generated with a variety of different sgRNA libraries.

\section{CRISPRcleanR preserves cell line essentiality profiles}

We next determined whether the correction performed by CRISPRcleanR alters the overall essentiality profile of a given cell line. For Project Score data, we checked the position of sets of top-depleted sgRNAs from uncorrected $\operatorname{logFCs}$ along the profiles of corrected sgRNA logFCs by means of precision/recall analysis (Fig. 4a, b). We observed a median area under the precision/recall curve (AUPRC) of 0.92 ( $\mathrm{min}=0.81$ for HCC-15, $\max =0.96$ for MDA-MB-436) for the top 50 depleted sgRNA, and a median AUPRC of 0.96 for the top 2500 depleted sgRNA $(\min =0.88$ for $\mathrm{HCC}-15, \max =0.98$ for MDA-MB-453). Considering that an experiment typically yields $\sim 6000$ sgRNAs called as significantly depleted with our library, this indicates that the CRISPRcleanR correction, while reducing false-positive rates, does not have an unwanted impact on the overall essentiality profile of a cell line.

To further assess the impact of CRISPRcleanR on gene essentiality profiles, we compared all genes with a significant gain or loss-of-fitness effect before and after CRISPRcleanR correction as this is the key phenotype measured in CRISPR-KO screens (Additional file 2: Figure S8). For Project Score data, we found CRISPRcleanR impacted on the significant loss/gain-of-fitness effect for a median of $1.98 \%$ of all screened genes. This included a median of $24.69 \%$ genes significantly detected as exerting an effect on cellular fitness (gain- or loss-of-fitness) and a median of $17.02 \%$ of loss-of-fitness genes. The vast majority (88\%) of these attenuated loss-of-fitness genes were composed of putatively false positive hits, involving genes which are not expressed (FPKM <0.05), located in $\mathrm{CN}$-amplified segments, prior known non-essential, or genes with a weak loss-of-fitness effect when compared to the whole set of genes called as loss-of-fitness in the uncorrected data (average logFC over the 4th quartile). For a very small number of genes (median $0.02 \%$ of genes, $n=28$ unique 


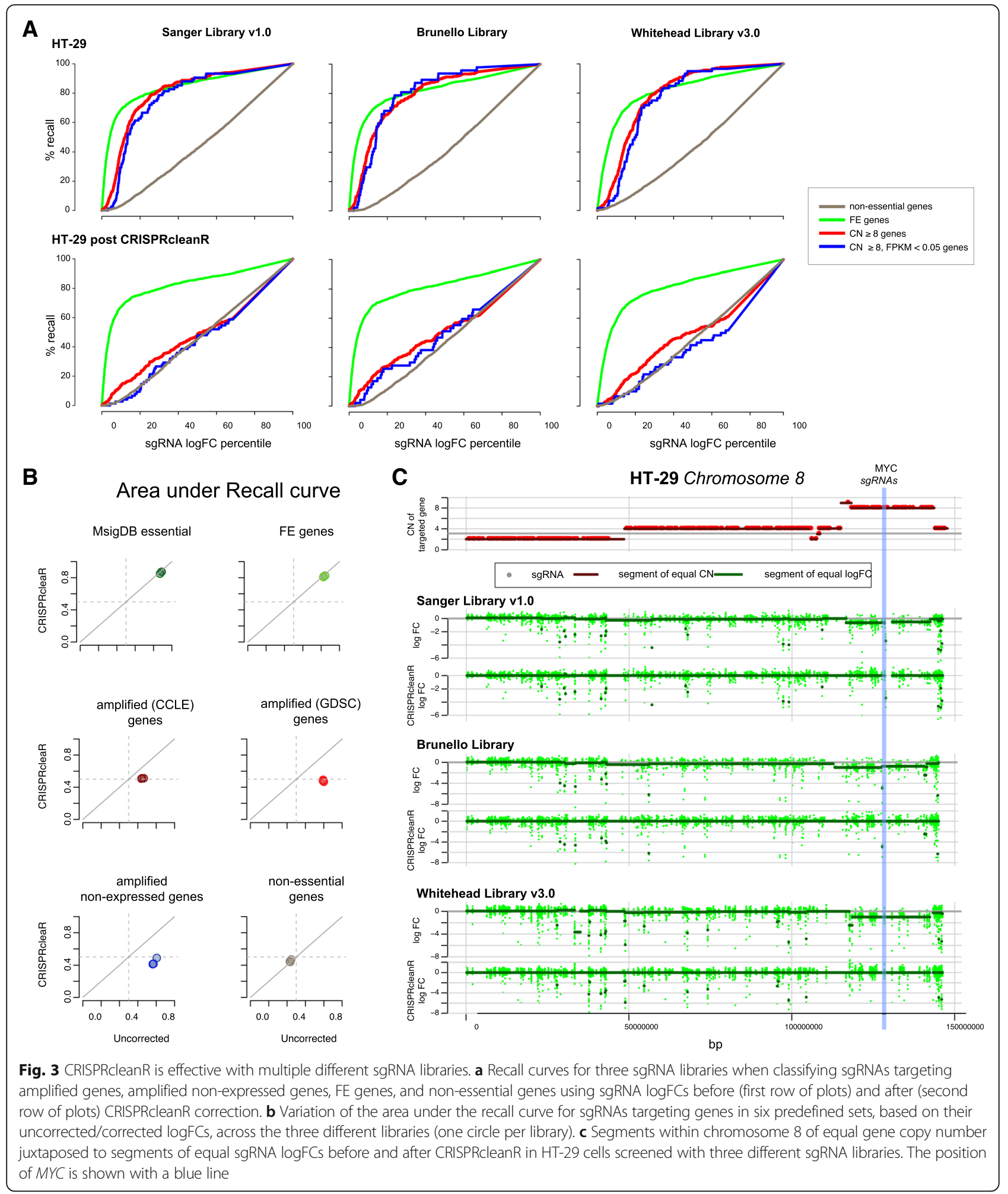

genes total) the post-correction fitness effect was opposite to that observed prior to the correction. A very similar effect on significant genes following CRISPRcleanR correction was observed for the Project Achilles data (Additional file 2: Figure S8). Thus, CRISPRcleanR preserves the overall essentiality profile present in a cell line and alters the significant fitness effects observed in the uncorrected data for only a minority of genes. Where correction occurs, the majority of instances involve likely putative false positive genes. 

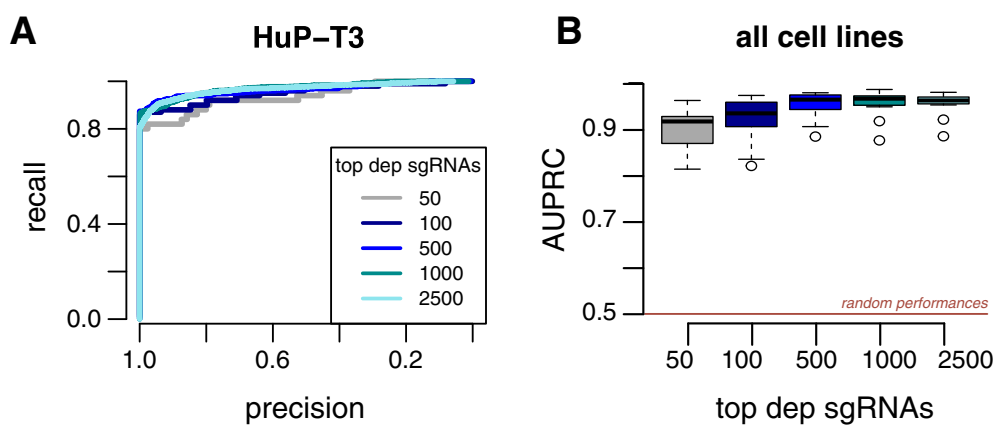

Fig. 4 CRISPRcleanR retains overall essentiality profiles. a Example precision/recall curves in HuP-T3 cells for the indicated number of top depleted sgRNAs after CRISPRcleanR correction, classified based on their un-corrected sgRNAs logFC rank position. b Area under the precision/recall curves defined as for A for all cell lines. Box-plots show the median, inter-quartile ranges and 95\% confidence intervals

\section{CRISPRcleanR corrects sgRNA counts to enable mean- variance modeling}

MAGeCK is a widely used computational tool to call gene depletion or enrichment in CRISPR-KO screens and is based on mean-variance modelling of median-ratio normalised sgRNA read-counts [22]. To make CRISPRcleanR compatible with mean-variance modeling approaches such as MAGeCK, we designed an inverse transformation to derive corrected sgRNA treatment counts from CRISPRcleanR corrected sgRNA logFC values. To benchmark our transformation, we compared results obtained from executing MAGeCK using normalised uncorrected and CRISPRcleanR corrected sgRNA counts by means of recall estimation when classifying predefined gene sets. The inverse transformation had an effect on both the mean and variance of the sgRNA counts, with the greatest impact on sgRNAs targeting genes in $\mathrm{CN}$-amplified regions, whose value was consistently shifted toward the corresponding value in the plasmid/control condition (Fig. 5a, b). Furthermore, we observed a strong reduction in recall when classifying sgRNAs targeting genes in biased regions (PicNic scores $\geq 8$ or GISTIC $\geq 2$ ), when considering as positive predictions the sgRNAs called significantly depleted by MAGeCK. The median reduction was $75 \%$ for $\mathrm{CN}$-amplified genes and $80 \%$ for $\mathrm{CN}$-amplified non-expressed genes at a $10 \% \mathrm{FDR}$, and $72 \%$ and $100 \%$ reductions at a $5 \%$ FDR (Fig. $5 \mathrm{c}$ and Additional file 1: Table S4). In contrast, the effect on the recall of $\mathrm{FE}$ and non-essential genes was negligible (median = $2.9 \%$ reduction) (Fig. $5 \mathrm{c}$ ). Thus, the reverse transformation post-correction enables the use of mean-variance modelling approaches such as MAGeCK for downstream calling of significant depletion or enrichment of genes.

\section{Robust detection of cancer dependencies following CRISPRcleanR}

Since a major application of CRISPR-KO screens is the accurate identification of genes essential for cellular fitness in defined molecular settings, we investigated the ability of
CRISPRcleanR to preserve the detection of expected cancer gene dependencies in individual cell lines. To perform a systematic analysis, we used CRISPRcleanR corrected sgRNA counts and a set of 64 cancer driver genes [27] which are modified by somatic mutation or $\mathrm{CN}$ amplification. We considered $\mathrm{CN}$ amplifications at the segment level (from [13]), thus including multiple genes in a segment.

Project Score cell lines included a total of 57 potential dependencies, involving a total of 29 cancer driver genes (9 mutated and 20 genes in amplified $\mathrm{CN}$ segments). Of these, we detected 21 dependencies prior to CRISPRcleanR correction (MAGeCK FDR $<10 \%$ ), and 16 of them (76\%) were preserved following CRISPRcleanR correction (Additional file 2: Figure S9 and Additional file 1: Table S5). Examples included SW48 carrying the EGFRg719s mutation associated with depletion of EGFR targeting sgRNA, and MDA-MB-453 carrying the PIK3CAh1047r mutation associated with depletion of PIK3CA targeting sgRNA (Fig. 6a).

CRISPRcleanR preserved the ability to selectively detect cancer dependencies involving amplified cancer driver genes. For example, $M Y C$ is amplified in the cell line HT-29 and sgRNAs targeting $M Y C$, as well as flanking genes, are reported as significantly depleted when using uncorrected logFCs (Fig. 6b). The logFC depletion is greater for $M Y C$ compared to other genes in this region. Following CRISPRcleanR correction, the sgRNAs targeting $M Y C$ remained significantly depleted, whereas those targeting the co-amplified flanking genes were no longer significant. A similar essentiality was selectively preserved post-CRISPRcleanR correction in an amplified region of chromosome 16 that contains ERBB2 in the NCI-H2170 cell line (Fig. 6c). Two of the dependencies attenuated post correction involved co-amplification of two driver genes; CDK12 co-amplified with ERBB2 in NCI-H2170 and CTTN co-amplified with CCND1 in MBA-MB-415 were no longer significant post correction. Similar results were found using the Project Achilles data with an overall retention rate of $80 \%$ (179 of 233) of dependencies post 
A

Targeted Genes
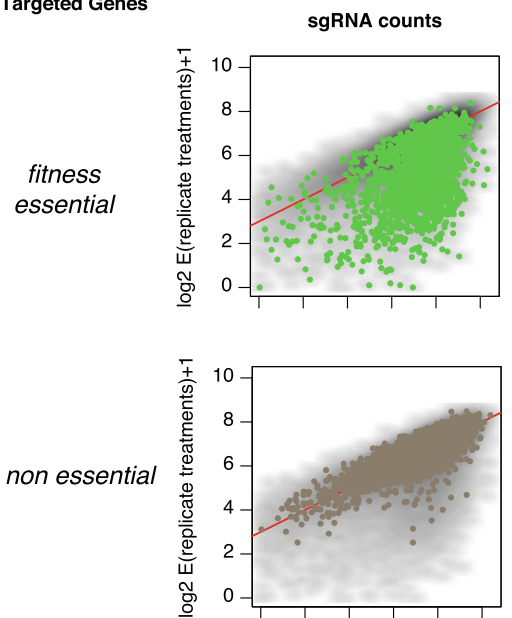

$\diamond E R B B 2$

$\diamond C C N D 1$
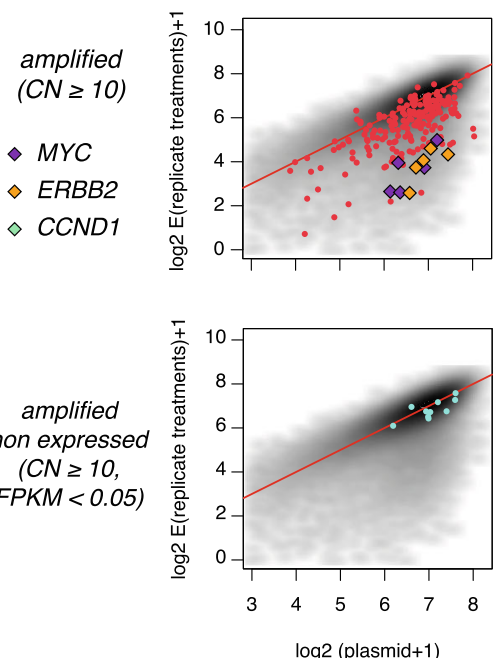

C
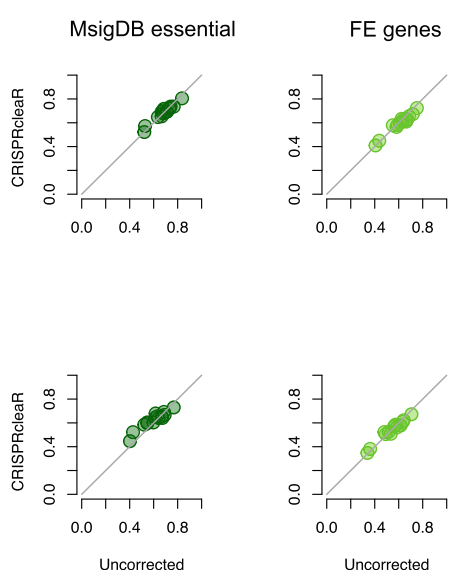

Fig. 5 (See legend on next page.)
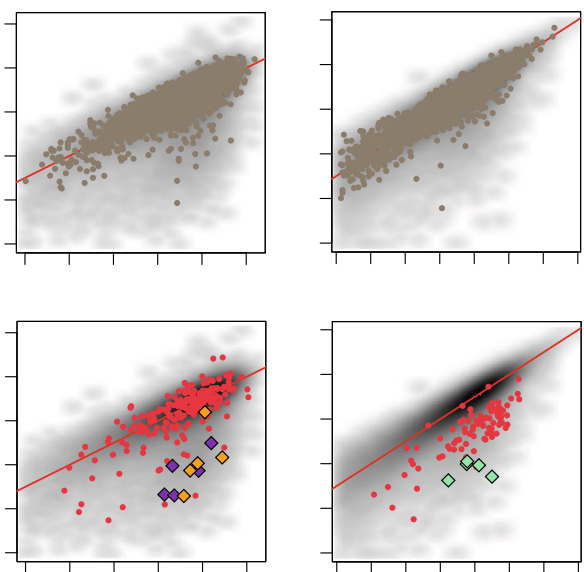

\section{B MDA-MB-415}
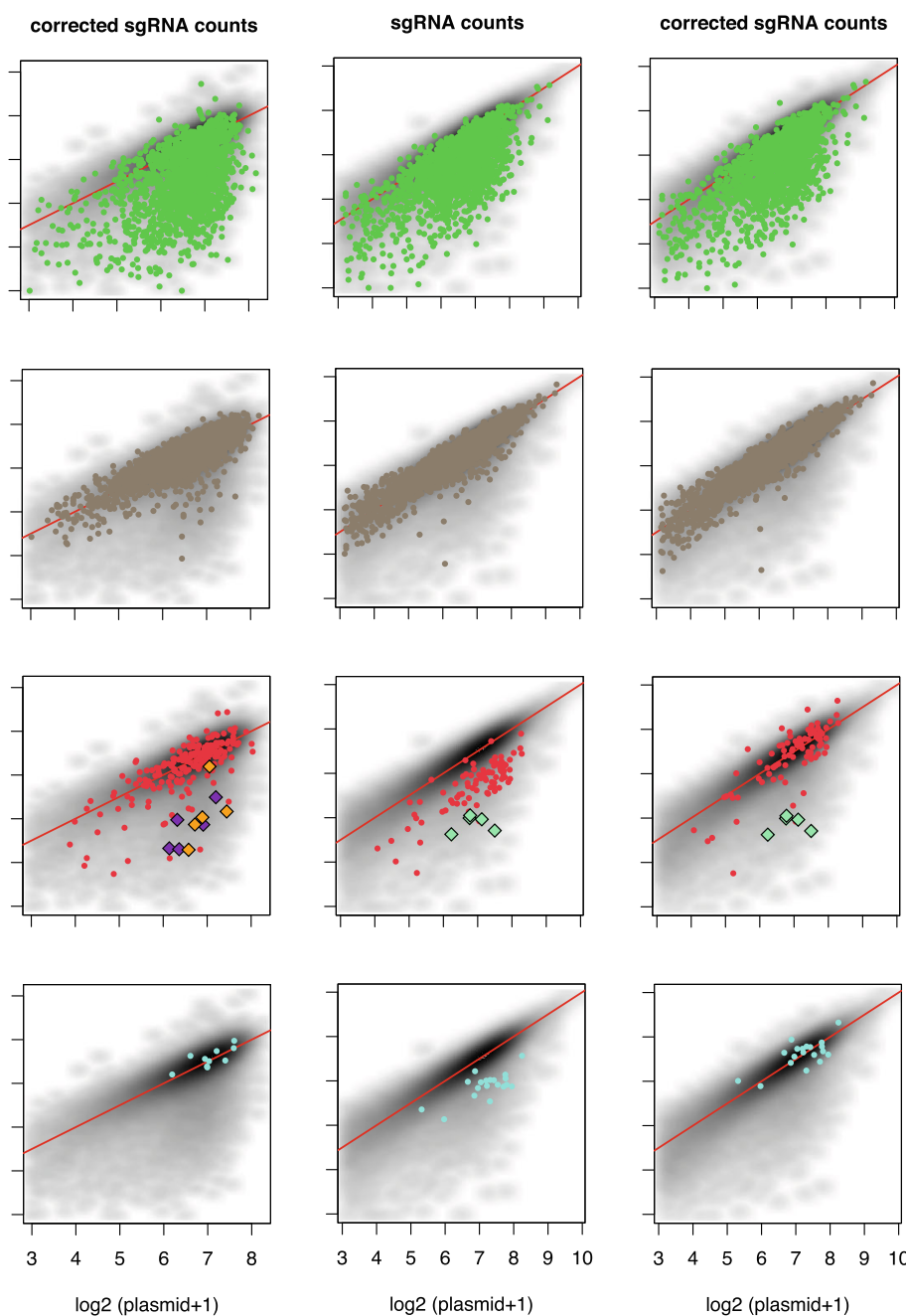

$\log 2$ (plasmid +1 )

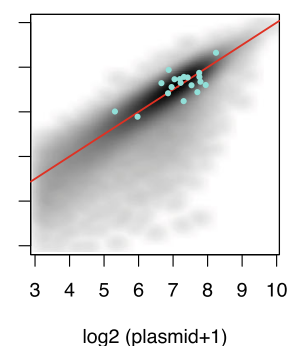

Recall at $10 \%$ MAGeCK FDR
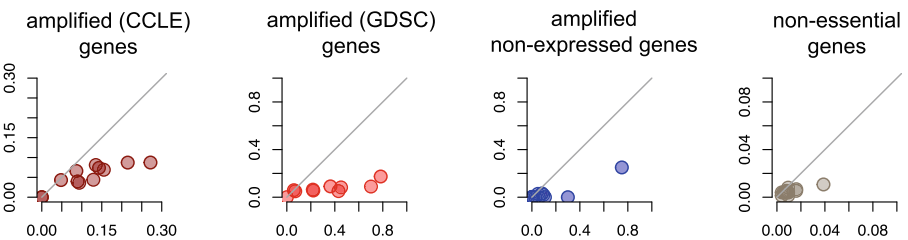

Recall at 5\% MAGeCK FDR
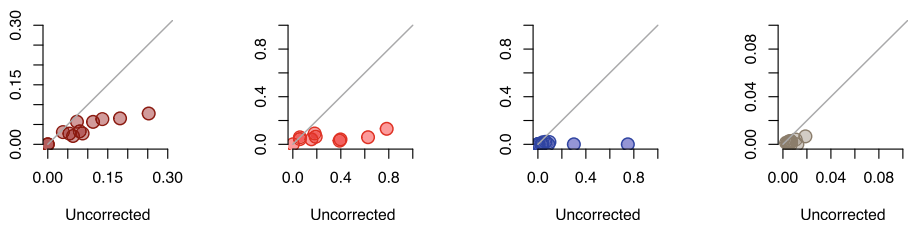
(See figure on previous page.)

Fig. 5 CRISPRcleanR corrected sgRNA counts and downstream analysis with MAGeCK. a and $\mathbf{b}$ Normalised counts of sgRNAs of the transfected libraries versus the control plasmid for FE and non-essential genes (first two rows of plots), CN amplified genes (third row) and CN non-expressed genes (fourth row), for two example cell lines before (first and third column) and after (second and fourth column) CRISPRcleanR correction. Essentialities for CN-amplified cancer driver genes such as MYC, ERBB2 and CCND1 are retained post correction. For the sake of readability only genes with at least 10 copies have been highlighted. c Comparison of recall using MAGeCK for sgRNAs targeting genes in six predefined gene sets when using as input CRISPRcleanR uncorrected and corrected sgRNAs counts

CRISPRcleanR correction (Additional file 2: Figure S9 and Additional file 1: Table S5). Of the attenuated dependencies, $41 \% \quad(n=44)$ involved genes co-amplified with another driver gene. In addition, we observed in both datasets a trend of increased significance (as measured by FDR) of detected dependencies post-correction. Overall, these results demonstrate that CRISPRcleanR allows for the accurate detection of cancer driver gene dependencies in CRISPR-KO datasets, including cancer genes residing within $\mathrm{CN}$-amplified regions.

\section{Code availability and overview}

CRISPRcleanR is implemented as an $\mathrm{R}$ [28] package and as an interactive Python package with full documentation, tutorials, built in datasets to reproduce the results in this manuscript, and is publically available ( $\mathrm{R}$ package: https://github.com/francescojm/CRISPRcleanR and Python package: https://github.com/cancerit/pyCRISPRcleanR). The Python implementation is dockerized making it platform independent and usable in cloud environments (https://dockstore.org/containers/quay.io/ wtsicgp/dockstore-pycrisprcleanr). CRISPRcleanR includes core functions for processing raw sgRNA count files for generating corrected sgRNA $\operatorname{logFC}$ values and corrected sgRNA counts for downstream analyses. CRISPRcleanR also includes functions to measure and visualise the extent and effect of the performed correction, the ability to detect $\mathrm{CN}$-amplified non-expressed genes (which can be used as positive controls), and classification performances for a priori known sets of essential/non-essential genes pre- and post-correction.

\section{Discussion}

In this study, we report CRISPRcleanR, a computational tool that detects genomic segments of gene-independent responses to CRISPR-KO in an unsupervised manner, and applies a segment-by-segment correction at the sgRNA-level for both fold-changes and read counts. The correction substantially reduces false-positive calls without altering true essentiality profiles and preserves known cancer gene dependencies within and outside of biased segments. CRISPRcleanR works on multiple genome-wide sgRNA libraries, and resulting corrected sgRNA $\log \mathrm{FC}$ and read counts are compatible with downstream analyses performed by methods such as BAGEL or MAGeCK to statistically assess screen hits.
CRISPRcleanR works efficiently irrespective of the sample size of the analysed dataset, even in single sample experiments.

Our motivation for developing CRISPRcleanR came from the observation that biases in gene essentialities observed in CRISPR-KO screens did not always show a linear correlation to their $\mathrm{CN}$ status, although biased segments are frequently associated with $\mathrm{CN}$ alteration. Additionally, in most of the cell lines analysed, variation in the mean $\operatorname{logFC}$ of segments with the same $\mathrm{CN}$ were often greater than those between segments with different $\mathrm{CN}$. Some cell lines showed greater bias in segments with lower CN. We even identified multiple instances of discontinuous bias on sgRNA logFCs within a particular CN segment, and biased responses within segments that are not $\mathrm{CN}$-amplified. These observations argue for the development of methods such as CRISPRcleanR, which are independent of $\mathrm{CN}$ values for the analysis of CRISPR-KO screening data, and indicate that biased responses are not solely due to the amount of DNA damage and may also be caused by additional factors, such as local genomic structural variation (as recently reported in [29]).

CRISPRcleanR detects biased segments using sgRNA-level $\log F C$ in an unsupervised manner, eliminating the requirement for cell line $\mathrm{CN}$ information. This simplifies the analysis and is advantageous when reliable $\mathrm{CN}$ information is not available for a cell line; for example, when using a newly derived cancer cell model. In addition, cancer genomes are dynamic and continuously evolving, causing genetic variation between different clones of the same cell line. Genetic drift may occur during prolonged in vitro cell culture, due to different growth conditions (e.G. media composition), or in response to selective pressure (e.g drug treatment) and genetic manipulation (e.g. gene-editing). Thus, the genomic heterogeneity of cancer cells, even within clones of the same cell line, may confound $\mathrm{CN}$-based correction methods when relying on pre-existing $\mathrm{CN}$ data, and negatively impact identification of gene essentialities. Furthermore, the performance of different copy number calling algorithms is variable and depends on the underlying genomic data available, and as a result this can be a further confounding factor when using $\mathrm{CN}$-based correction methods. CRISPRcleanR overcomes these limitations by effectively correcting for biases in CRISPR-KO screens without requiring additional information about 


\section{A}

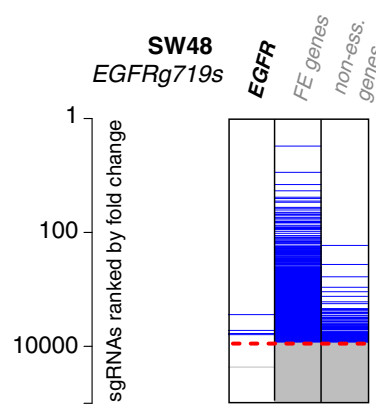

EGFR

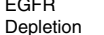

Depletion
FDR $=0.0008$

MDA-MB-453

PIK3CAh1047r है के हो के
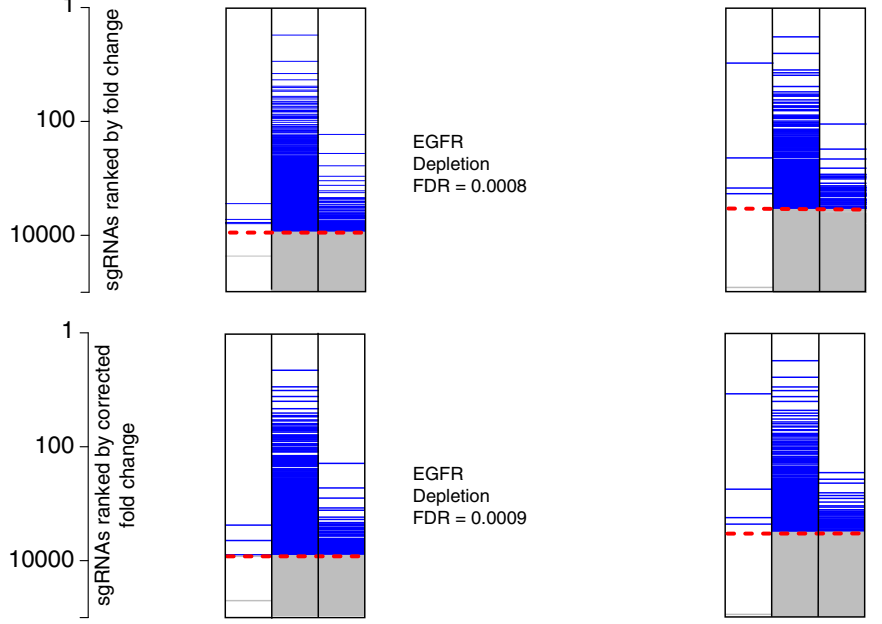

PIK3CA

Depletion
FDR $=0.025$

EGFR

Depletion

FDR $=0.0009$

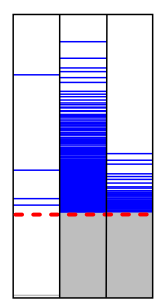

PIK3CA

Depletion
FDR $=0.007$

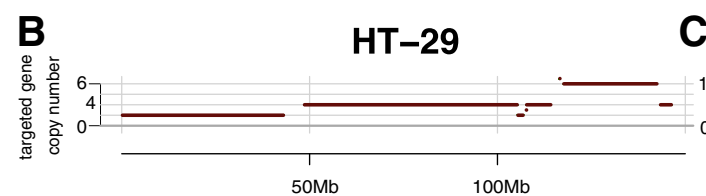

C $\quad \mathrm{NCl}-\mathrm{H} 2170$

Chr8 \begin{tabular}{|l|l|l|l|l|l|l|l|l|}
\hline & Chr17 \\
\hline
\end{tabular}
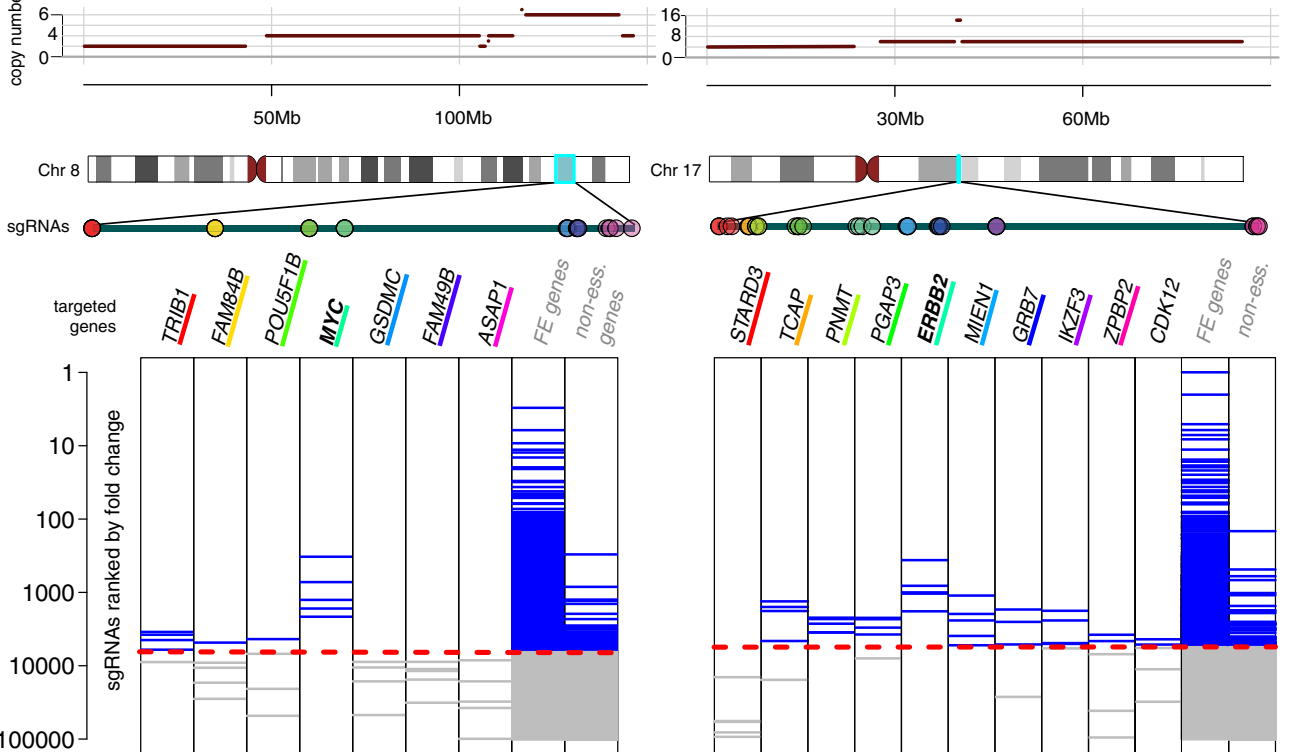

$30 \mathrm{Mb}$

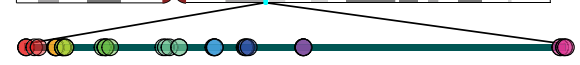

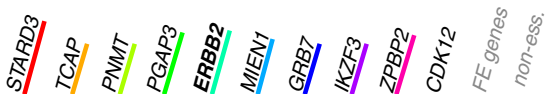

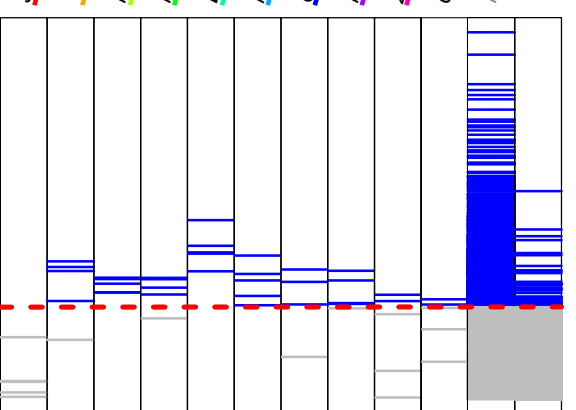

- $5 \%$ FDR
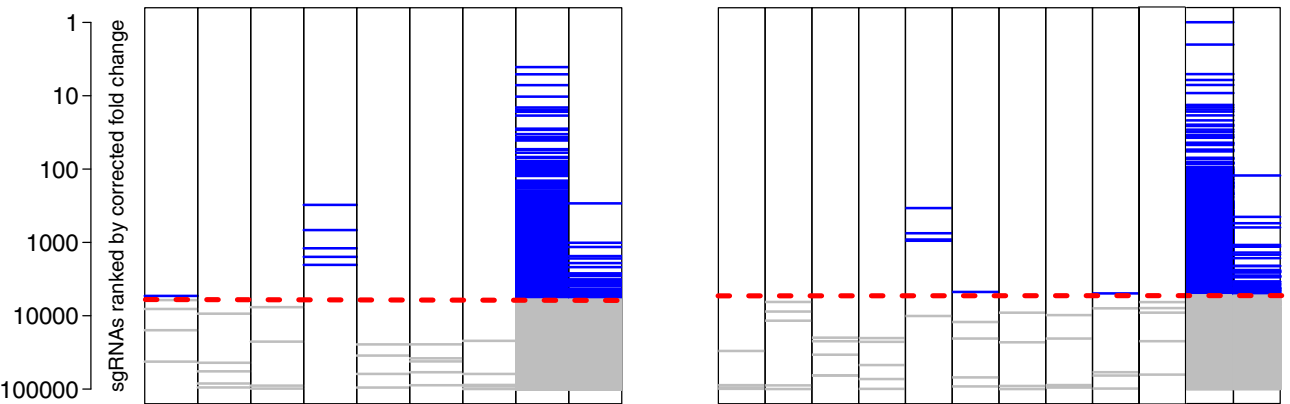

Fig. 6 (See legend on next page.) 


\section{(See figure on previous page.)}

Fig. 6 CRISPRcleanR enables detection of cancer gene dependencies. a Detection of EGFR and PIK3CA dependencies at the level of targeting sgRNAs in mutant cancer cell lines. Rank position of sgRNAs targeting the indicated genes before (top) and after (bottom) CRISPRcleanR correction. FE and non-essential genes are shown for comparison. b A CN-amplified region of chromosome 8 in HT-29 cell line including MYC and 3 surrounding up-streaming/down-streaming genes. Expanded view of sgRNAs targeting MYC and its surrounding genes, with each gene identified by a different colour. The heatmaps (first 7 columns) show ranked positions of the sgRNAs targeting the 7 considered genes (blue bars) before (top heatmap) and after (bottom heatmap) CRISPRcleanR correction. The last two columns show rank positions for the sgRNAs targeting FE genes (second last column) and non-essential genes (last column). c Same as for B but considering a region on chromosome 16 in the NCIH2170 cell line, including ERBB2 and four flanking upstreaming/downstreaming genes and CDK12

the cell models screened, and without making assumptions about the underlying cause of bias.

\section{Conclusion}

CRISPRcleanR is a flexible tool implemented as $\mathrm{R}$ and Python packages to correct gene-independent bias found in whole-genome CRISPR-KO screens in an unsupervised manner at a single sample level. CRISPRcleanR facilitates the analysis of CRISPR-KO screens in cancer cells to identify essential genes.

\section{Methods}

Plasmids, cell lines and reagents

Cells were maintained in culture media as indicated in Additional file 1: Table $\mathrm{S} 1$ in a $5 \% \mathrm{CO}_{2}$ humidified incubator at $37{ }^{\circ} \mathrm{C}$. The plasmids used in this study were from the mutagenesis toolkit described in [6] and are available through Addgene (Cas9-68,343; CRISPR sgRNA library 67,989). Plasmids were packaged using the Virapower (Invitrogen) system as per manufacturer's instructions.

\section{Genome-wide mutant library and screen}

Cells were first transduced with lentivirus carrying Cas9 in T75 flasks at $\sim 80 \%$ confluence in the presence of polybrene $(8 \mu \mathrm{g} / \mathrm{ml})$. The following day, lentiviral containing medium was replaced with complete medium. Blasticidin selection was started on day 4 post transduction at a concentration determined from a titration in the parental cell line. Cas9 activity was assessed following selection using the Cas9 functional assay as described in [6] and a cut-off of $80 \%$ activity was applied (median $=89 \%$ activity across all cell lines). Cas9-expressing cells were maintained in blasticidin prior to transduction with the sgRNA library. Transduction with sgRNA library was carried out at $\sim 80 \%$ confluency with $3.3 \times 10^{7}$ cells in T150 or T525 (triple layer) flasks, depending on cell size and surface area required, in technical triplicates. Cells were transduced with a predetermined viral amount that gives rise to $30 \%$ transduction, measured by BFP expression by cytometry, to ensure approximately 1 viral particle entering each cell based on a Poisson distribution model. Based on these initial cell numbers and transduction efficiency, the coverage of the sgRNA library (i.e. the number of cells containing each sgRNA) in each replicate was $100 \times$. Puromycin selection commenced at day 4 to select for cells that had successful lentiviral integration. Actual library transduction efficiency and puromycin selection was analysed using flow cytometry before and after puromycin selection, respectively. A minimum number of $5.0 \times 10^{7}$ cells were maintained at all times to ensure library representation was maintained. The cells were harvested 14 days post transduction and dry pellets were stored at $-80^{\circ} \mathrm{C}$.

Extraction of genomic DNA, PCR amplification of sgRNAs and Illumina sequencing of sgRNAs were carried out as described previously $[3,6]$. The number of reads for each sgRNA was determined using a script developed in-house.

\section{Data pre-processing and availability}

sgRNA counts from both Project Score and Project Achilles (downloaded from: https://depmap.org/ceres/) were normalised assembling one batch per cell line, including the read counts from the matching library plasmid and all final read counts replicates, with a median-ratio method [30] to adjust for the effect of library sizes and read count distributions, after filtering out sgRNAs with less than 30 reads in the plasmid. Depletions/enrichments for individual sgRNAs were quantified as $\log _{2}$ ratio between post library-transfection read-counts and library plasmid read-counts. Finally, sgRNAs were averaged across replicates. This was performed executing the ccr.NormfoldChanges function of the CRISPRcleanR R package.

\section{Transcriptional and copy number data}

Genome-wide substitute reads with fragments per kilobase of exon per million reads mapped (FPKM) for the 15 cell lines considered in this study were derived from the dataset described in [31]. Genome-wide gene level copy number data, derived from PicNic analysis of Affymetrix SNP6 segmentation data (EGAS00001000978) for the cell lines in the Genomics of Drug Sensitivity 1000 (GDSC1000) cancer cell line panel [13], were downloaded from the GDSC data portal (dataset version: July 4th 2016), http://www.cancerRxgene.org. This dataset is also available at http://ftp.sanger.ac.uk/pub/project/cancerrxgene/releases/release-6.0/Gene_level_CN.xlsx. For each gene, the minimum copy number of any genomic segment containing coding sequence was considered. 
Additionally, gene level Gistic [25] scores obtained by processing Affymetrix SNP array data in the Cancer Cell Line Encyclopaedia [32] repository were downloaded from cBioPortal [33] (http://www.cbioportal.org/ study?id=cellline_ccle_broad\#summary).

Analysis of gene-independent responses in cancer cell lines For each cell line, segments of equal $\mathrm{CN}$ were identified by using $\mathrm{CN}$ data from the GDSC data portal $[13,14]$ (as detailed below), and assigned a mean-logFC value by averaging across all of the sgRNAs targeting a segment. A CN bias starting point was computed for each cell line as the copy number value $n>2$ such that statistically significant differences, as quantified by a Welch's t-test, were observable between the mean-logFCs of segments of $n \mathrm{CNs}$ and those of segments of $2 \mathrm{CN}$. A CN bias critical point was computed for each cell line as follows. For each $\mathrm{CN}$ value $n=3, \ldots$, $m$-1 (with $m=$ maximal segment $\mathrm{CN}$ value observed in the cell line under consideration), two univariate linear models were fitted, considering segment $\mathrm{CN}$ values as observations of the independent variable and the corresponding average segment mean-logFCs as those of the dependent one. The first model $P(n)$ was fitted using $C N$ values in $\{2, \ldots, n\}$ and corresponding average segment mean-logFCs, while the second one $L(n)$ was fitted using $\mathrm{CN}$ values in $\{n+1, \ldots, m\}$ and corresponding average segment mean-logFCs. The bias critical point was then defined as the value $n$ providing the large absolute difference between the slopes of the corresponding fitted models $P(n)$ and $L(n)$.

\section{Calling significantly depleted sgRNAs and genes based on log fold-changes}

All sgRNA were ranked by average logFCs derived from screening an individual cell line. This ranked list was used to classify sgRNAs targeting genes from two gold-standard reference sets of FE and non-essential genes [15, 21]: from now the essential-sgRNAs $(E)$ and the non-essential-sgRNAs $(N)$. For each rank position $k$, a set of predictions $P(k)=\{s \in E \cup N: \varrho(s) \leq k\}$, with $\varrho(s)$ indicating the rank position of $s$, was assembled and corresponding Precision (or Positive Predicted Value, $P P V(k))$ was computed as:

$$
P P V(k)=|P(k) \cap E| /|P(k)| .
$$

Subsequently the largest rank position $k^{*}$ corresponding to a 0.95 Precision (equivalent to a False Discovery Rate $(F D R)=0.05)$ was determined as.

$$
k^{*}=\max _{k=1}^{N}\{1-P P V(k) \leq 0.05\} .
$$

Finally, a 5\% FDR $\log \mathrm{FCs}$ threshold $F^{*}$ was determined as the $\operatorname{logFCs}$ of the sgRNAs $s$ such that $k(s)=k^{*}$, and all the $\operatorname{sgRNAs}$ of the entire library with a $\operatorname{logFC}<F^{*}$ were considered significantly depleted at this FDR level.

To call depletion significance at a gene level, the same procedure was followed but averaging $\operatorname{logFCs}$ of sgRNAs targeting the same gene prior to the analysis, and considering ranks and positive/negative sets of genes instead of sgRNAs.

For the follow up analyses on the effect of correcting sgRNA treatment counts (computed as detailed below) we used the test function of the MAGeCK python package, indicating none as the value of the parameter specifying the normalisation method to use prior to the analysis, as a median-ratio normalisation was already applied to the analysed count files prior CRISPRcleanR correction.

\section{Receiver operating characteristic analyses}

Across the different analyses, standard ROC indicators were computed considering as prediction sets significantly depleted sgRNAs (or genes) at a fixed level of 5\% FDR (computed as detailed in the previous section or output by the MAGeCK tool), or genome-wide profiles of essentiality (as ranked lists of sgRNA logFCs, in some instances averaged on a per gene basis) to compute overall indicator curves, and using different positive/negative control sets (detailed below). To this aim, we made use of functions included in the pROC R package [34].

For the positive controls, sets of a priori essential genes were assembled by downloading relevant gene signatures from the MSigDB [16] (Additional file 1: Table S6). A list of ribosomal protein gene was derived from [17]. The consensual signatures resulting from this curation are available as individual data objects in the CRISPRcleanR $\mathrm{R}$ package.

\section{Segmentation analysis and logFC correction}

Genome-wide essentiality profiles in the form of lists of sgRNAs $\operatorname{logFC}$ were sorted according to the genomic coordinates of the individual sgRNAs (library annotation and coordinates derived from [6]) using the function ccr.logFCs2chromPos of the CRISPRcleanR $\mathrm{R}$ package. Then, a circular binary segmentation algorithm $[18,19]$ was applied using the ccr.GWclean function of the CRISPRcleanR R package, with a significance threshold to accept change-points $p=0.01,10,000$ permutations for $p$-value computation, a minimal number of 2 markers per region, and making use of the function segment from the DNAcopy R package [20] with other parameters set to default values.

Subsequently, sgRNA included in a segment had their $\operatorname{logFCs}$ mean-centered (across that segment) if collectively targeting at least $n=3$ different genes, without pre-filtering any essential gene (differently from the sliding window approach used in [9]). This correction assumes that the true signal of loss/gain-of-fitness effect exerted by knocking-out 
a CN amplified gene sums up to a possible gene-independent impact on cellular fitness induced by targeting with CRISPR-Cas9 the chromosomal segment where that gene resides. By subtracting the logFCs mean to the sgRNA in the same detected biased segment, the gene-independent effect is flattened letting true fitness signals emerge. The possibility of using a median-based centering as a more robust alternative when the data is particularly noisy and/or many outliers are present (verifiable through a preliminary inspection of the uncorrected $\log \mathrm{FCs}$ ), for example due to dysfunctional or especially toxic sgRNAs, is also present in the implementation of CRISPRcleanR.

The minimal number $n$ of targeted genes that a biased segment should contain in order to be corrected was adaptively determined by executing different trials of segments' detection and correction varying $n \in\{2,3,5,10\}$ and excluding/not-excluding from the analysis sets of a priori known essential genes assembled from MSigDB (as detailed in the previous section), collectively the filter set. Removing the filter set from a reference set of the FE genes yielded a test set. Areas under the recall curve (AURCs) were then computed evaluating the classification performances using as positive controls the test set, $\mathrm{CN}$ amplified genes, and $\mathrm{CN}$ amplified non-expressed genes (determined for each cell line) were then computed, across each trial using targeting sgRNAs' logFCs before/after correction. For each of the positive control sets, reduction of recall (recall) were computed by comparing AURCs obtained before/after CRISPRcleanR correction.

Results showed that $n=3$ provided the largest reduction of recall (Additional file 2: Figure S5) of $\mathrm{CN}$ amplified and $\mathrm{CN}$ amplified non-expressed genes, and the lowest reduction of recall of the test set. Most importantly, this was observed invariantly with respect to removing/not-removing the filter set prior the analysis. As a conclusion, all the corrections presented in this manuscript were executed with this setting $(n=3$ and without pre-filtering any gene). CRISPRcleanR package uses these settings by default, although offering to the user the possibility of changing them.

\section{Comparison of results across different libraries}

Data from the mutagenesis of the HT-29 cell lines with the Brunello and Whitehead libraries were downloaded from the supplementary material of [24] and processed as described in the section Data pre-processing and availability. Correction outcomes were computed as detailed in Receiver Operating Characteristic analyses.

\section{Correction of sgRNA counts}

We derived CRISPRcleanR corrected treatment count values for individual experiment technical replicates from the corresponding CRISPRcleanR corrected
sgRNAs' logFCs. To this aim, for each individual sgRNA, we first compute a CRISPRcleanR corrected treatment count averaged-across-replicate (first 7 formulas below), then we computed corrected treatment counts for individual replicates from this averaged value partitioning it across replicates proportionally to original (uncorrected) count values.

Formally, for each individual single guide RNA, a corrected treatment count $t_{i}$ was computed observing that:

$$
N=E\left(\log _{2} \frac{t_{i}}{c}\right)
$$

with $N=$ CRISPRcleanR corrected $\operatorname{logFC}$ for the sgRNA under consideration, $i=1, \ldots, n$, where $n=$ number of treatment replicates, and $c=$ counts of the sgRNA in the plasmid, and $E$ indicates the mean function.

This implies

$$
\begin{aligned}
& N=\frac{\sum_{i=1}^{n} \log _{2}\left(t_{i} / c\right)}{n} \\
& \Rightarrow n N=\sum_{i=1}^{n} \log _{2} t_{i}-\sum_{i=1}^{n} \log _{2} c=\sum_{i=1}^{n} \log _{2} t_{i}-n \log _{2} c \\
& \Rightarrow n N+n \log _{2} c=\sum_{i=1}^{n} \log _{2} t_{i} .
\end{aligned}
$$

Assuming, for simplicity that all the $t_{i}$ are the same $(=t)$,

$$
\begin{aligned}
& n N+n \log _{2} c=n \log _{2} t_{i} \\
& \Rightarrow 2^{N+\log _{2} c}=t \\
& \Rightarrow t=c 2^{N}=E(t) .
\end{aligned}
$$

To derive the corrected counts for the individual replicates (which are obviously different from each other) from their mean, we keep constant the proportions seen in the uncorrected counts with respect to the sum of the counts across replicates:

$$
\begin{aligned}
& E(t)=\frac{\sum_{i=1}^{n} t_{i}}{n} \\
& \Rightarrow n E(t)=\sum_{i=1}^{n} t_{i} \\
& \Rightarrow t_{i}=n E(t) \frac{t_{i}^{*}}{T^{*}}=n c 2^{N} \frac{t_{i}^{*}}{T^{*}}
\end{aligned}
$$

where $t_{i}^{*}$ is the count of the sgRNA under consideration before correction in the $i$-th replicate and $T^{*}$ is their overall sum across replicates. 
CRISPRcleanR performances with respect to data quality Cell lines from Project Achilles were grouped into 10 equidistant bins based on the quality of the corresponding profiles of gene essentiality, in increasing order. Data quality was quantified by the recall at $5 \%$ FDR for MSigDB [16] essential genes based on uncorrected fold-change rank positions. For each bin a variation of Recall pre/ post-CRISPRcleanR correction was quantified, for 9 predefined gene sets, encompassing prior known essential/ non-essential genes, copy number amplified genes and non expressed genes, as detailed in the previous sections.

\section{Evaluation of CRISPRcleanR correction on fitness gene calling}

Gain/loss-of-fitness effect false discovery rate (FDR) scores were obtained by applying MAGeCK before/after CRISPRcleanR correction on the sgRNA counts from Project Score and Project Achilles. Percentages of attenuated fitness genes were computed as the ratio of genes with a significant gain/loss-of-fitness FDR (fitness genes), from the analysis of the uncorrected sgRNAs but not from the analysis of the corrected ones, with respect to the whole set of screened genes or the set of fitness genes detected in the uncorrected data, respectively. Percentages of distorted fitness genes were computed as the ratio of fitness genes detected in the uncorrected data which where still detected as fitness genes in the corrected data but with an opposite effect. Similar ratios were computed for attenuated/distorted loss-of-fitness and gain-of-fitness genes individually. The loss-of-fitness genes attenuated post-correction were further partitioned sequentially into the following disjoint sets across cell lines: non-expressed (with an FPKM < 0.05), copy number amplified (with a Gistic score $>1$ or a PicNic copy number value $>2$ ), prior-known non-essential (according to [15]), mild-phenotype (with a depletion logFC in the uncorrected data, averaged across targeting sgRNAs, falling over the 4th quartile of the logFCs of all the loss-of-fitness genes). Only cell lines with good quality data (recall for essential genes from [15] at 5\% FDR > 0.5 ) and all data type (GISTIC and PicNic copy number, and basal expression FPKMs) available were included in this analysis.

\section{Retention of cancer driver gene dependencies following CRISPRcleanR correction}

We performed a systematic unbiased case-by-case probing of putative oncogene addictions, by evaluating how corresponding dependencies are detected prior/post CRISPRcleanR correction, using data from Project Score and Project Achilles. From a list of 64 high confidence oncogenes [27], we considered those harbouring a cancer driver event (CDE), i.e. a cancer driver somatic mutation or a $\mathrm{CN}$ amplification as defined in [13], in at least one cell line of the two considered panels. For the Project Achilles, the analysis was restricted to 239 cell lines with genomic data available in [13]. The considered $\mathrm{CN}$ amplifications were at the chromosomal segment level and many of them included more than one oncogene. For each CDE observed in a given cell line, we then compared the loss/ gain-of-fitness effect of the involved oncogene(s) observed prior/post-CRISPRcleanR in that cell line, quantified as MAGeCK FDRs. For Project Score, this resulted into 57 tested dependencies involving 29 CDEs (9 mutations and 20 CNAs encompassing multiple genes on the same segments). For Project Achilles, this resulted into 507 tested dependencies: 37 CDFEs (26 mutations and 11 CNAs encompassing multiple genes on the same segments).

\section{Additional files}

Additional file 1: Table S1. Project Score cell lines included in the study with annotations and screening description. Table S2. Quantification of copy number-associated bias before and after CRISPRcleanR correction. Table S3. Recall reduction following CRISPRcleanR correction across control gene-sets and cell lines. Table S4. Recall reduction post CRISPRcleanR correction across controls (mean-variance modeling). Table S5. Cancer driver gene dependencies following CRISPRcleanR correction. Table S6. List of gene signatures downloaded from MSigDB and used as positive controls. (ZIP 6330 kb)

Additional file 2: Figure S1. CRISPR-KO screening data quality assessment. (A) Average correlation between sgRNAs read-count replicates across cell lines. (B) Receiver operating characteristic (ROC) curve obtained from classifying fitness essential (FE) and non-essential genes based on the average logFC of their targeting sgRNAs. An example cell line OVCAR-8 is shown. (C) Area under the ROC (AUROC) curve obtained for cell lines from classifying FE and non-essential genes based on the average logFC of their targeting sgRNAs. (D) Recall for sets of a priori known essential genes from MSigDB and from literature when classifying FE and non-essential genes across cell lines (5\% FDR). Each circle represents a cell line and coloured by tissue type. Box and whisker plots show median, inter-quartile ranges and 95\% confidence intervals. (E) Genes ranked based on the average logFC of targeting sgRNAs for OVCAR- 8 and enrichment of genes belonging to predefined sets of a priori known essential genes from MSigDB, at an FDR equal to $5 \%$ when classifying FE (second last column) and non-essential genes (last column). Blue numbers at the bottom indicate the classification true positive rate (recall). Figure S2. Assessment of copy number bias before and after CRISPRcleanR correction across cell lines. sgRNA logFC values before and after CRISPRcleanR for eight cell lines are shown classified based on copy number (amplified or deleted) and expression status. Copy number segments were identified using Genomics of Drug Sensitivity in Cancer (GDSC) and Cell Line Encyclopedia (CCLE) datasets. Box and whisker plots show median, inter-quartile ranges and $95 \%$ confidence intervals. Asterisks indicate significant associations between sgRNA LogFC values (Welchs t-test, $p<0,005)$ and their different effect sizes accounting for the standard deviation (Cohen's D value), compared to the whole sgRNA library. Figure S3. CNassociated effect on sgRNA logFC values in highly biased cell lines. For 3 cell lines, recall curves of non-essential genes, fitness essential genes, copy number (CN) amplified and CN amplified non-expressed genes obtained when classifying genes based on the average logFC values of their targeting sgRNAs. Figure S4. Assessment of CN-associated bias across all cell lines. LogFC values of sgRNAs averaged within segments of equal copy number (CN). One plot per cell line, with CN values at which a significant differences (Welchs t-test, $p<0.05$ ) with respect to the logFCs corresponding to $\mathrm{CN}=2$ are initially observed (bias starting point) and start to significantly increase continuously (bias critical point). CN-associated bias is shown for all sgRNA, when excluding FE genes and histones, and for non-expressed genes only. Box and whisker plots show median, inter-quartile ranges and 95\% confidence intervals. Figure S5. CRISPRcleanR correction varying the minimal number of genes required and the effect of fitness essential genes. Recall reduction of (A) 
amplified or (B) amplified not-expressed genes versus that of fitness essentia and other prior known essential genes, when comparing CRISPRcleanR correction varying the minimal number of genes to be targeted by sgRNA in a biased segment (default parameter is $n=3$ ). Similar results were observed when performing the analysis including or excluding known essential genes. Figure S6. CRISPRcleanR performances across 342 cell lines from an independent dataset. Recall at 5\% FDR of predefined sets of genes based on their uncorrected or corrected logFCs (coordinates on the two axis) averaged across targeting sgRNAs for 342 cell lines from the Project Achilles. Figure S7. CRISPRcleanR performances in relation to data quality. The impact of data quality on recall at $5 \%$ false discovery rate (FDR) assessed following CRISPRcleanR correction for predefined set of genes. Project Achilles data ( $n=342$ cell lines) was binned based on the quality of uncorrected essentiality profile. This is obtained by measuring the recall at 5\% FDR for predefined essential genes (from the Molecular Signature Database) and grouping the cell lines in 10 equidistant bins ( 1 lowest quality and 10 highest quality) when sorting them based on this value. Recall increment for fitness essential genes was greatest for the lower quality data, indicating that CRISPRcleanR can improve true signal of gene depletion in low quality data. Figure S8. Minimal impact of CRISPRcleanR on loss/gainof-fitness effects. (A) The percentage of genes where the significance of their fitness effect (gain- or loss-of-fitness) is altered after CRISPRcleanR for Project Score and Project Achilles data. The upper row shows correction effects for all screened genes and the lower row for the subset of genes with a significant effect in the uncorrected data. Each dot is a separate cell line. Blue dots indicate the percentage of genes where significance is lost or gained post correction. Green dots indicate the percentage of genes where the fitness effect is distorted and the effect is opposite in the uncorrected data. (B) The majority of the loss-of-fitness genes impacted by correction are putative false positive effects affecting genes which are either not-expressed (FPKM < 0.5), amplified, known non-essential, or exhibit a mild phenotype in the screening data. (C) Summary of overall impact of CRISPRcleanR on fitness effects following correction when considering data for all cell lines. The colors reflect the percentage of genes with a loss-of-fitness, no phenotype or gain-of-fitness effect which are retained in the corrected data. Figure S9. CRISPRcleanR retains cancer driver gene dependencies in Project Score and Achilles data. (A) Each circle represents a tested cancer driver gene dependency (mutation or amplification of a copy number segment) and the statistical significance using MaGeCK before (x-axis) and after (y-axis) CRISPRcleanR correction, across the two screens. Plots in the first row show depletion FDR values pre/post-correction, whereas those in the second row show depletion FDR values pre-correction and enrichment FDR values postcorrection. (B) Details of the tested genetic dependencies and whether they are shared before and after CRISPRcleanR correction at two different thresholds of statistical significance ( 5 and $10 \%$ FDR, respectively for 1 st and 2 nd row of plots). The third row indicates the type of alteration involving the cancer driver genes under consideration and the total number of cell lines with an alteration. (ZIP $191 \mathrm{~kb}$ )

\section{Abbreviations}

AUPRC: Area Under the Precision/Recall Curve; AURC: Area Under the Overall Recall Curve; AUROC: Area Under the Receiver Operating

Characteristic Curve; CCLE: Cancer Cell Line Encyclopedia; CN: Copy Number; CRISPR-KO: CRISPR Knock-Out; DSB: Double Strand Break; FE: Fitness Essential; FPKM: Fragments Per Kilobase of Exon per Million reads Mapped; GDSC: Genomics of Drug Sensitivity in Cancer; logFC: Log Fold Change: MsigDB: Molecular Signature Database; ROC: Receiver Operating Characteristic; sgRNA: Single guide RNA; TPR: True Positive Rate

\section{Acknowledgements}

We thank David R. Wille, Vivek lyer, Leopold Parts, Felicity Allen, Gabriele Picco, and Ultan McDermott for a number of insightful discussions. We thank Giuseppe lorio for a number of acute and critical observations on the mathematics underlying this study.

\section{Funding}

This work was supported by OpenTargets (015), CRUK (C44943/A22536), SU2C, (SU2C-AACR-DT1213), the Wellcome Trust (102696) and Wellcome Sanger Institute core funding (206194).

\section{Availability of data and materials}

All data generated or analysed during this study are included in this published article and its supplementary information files. sgRNAs read count files analysed in this study are available for the HT-29, EPLC-272H, and A2058 cell lines as external data in the CRISPRcleanR R package, and they are used in the package vignette and documentation examples. sgRNA read counts for all cell lines used in this study have been deposited in BioStudies (https:// www.ebi.ac.uk/biostudies/, Accession number S-BSST79, dataset direct link: https://www.ebi.ac.uk/biostudies/studies/S-BSST79).

\section{Authors' contributions}

Fl conceived the study, designed algorithms, processed data, performed validation tests, implemented and documented the R package, wrote and revised the manuscript; FMB contributed to the design of the study, performed screens and related experiments, curated data, contributed to documenting the $\mathrm{R}$ package, wrote and revised the manuscript; EG contributed ideas for the design of the study, curated data, revised the manuscript; SGB wrote python implementation of R package; EC pre-processed data from the Project Achilles; RS tested python implementation; CMB, RA, RP, PW, SH performed screens and related experiments; APB supervised the python implementation, ES contributed ideas for the design of the study, co-supervised it and revised the manuscript; JSR contributed ideas for the design of the study and supervised it, revised the manuscript; KY conceived the study and co-supervised it, designed the sgRNA library and the experimental setting of the screen, wrote and revised the manuscript; MJG conceived the study, designed the experimental setting of the screen, wrote and revised the manuscript, supervised the study. All authors read and approved the final manuscript.

Ethics approval and consent to participate

Not applicable.

\section{Consent for publication}

Not applicable.

Competing interests

E.A.S. is an employee of GlaxoSmithKline.

\section{Publisher's Note}

Springer Nature remains neutral with regard to jurisdictional claims in published maps and institutional affiliations.

\section{Author details}

${ }^{1}$ European Molecular Biology Laboratory - European Bioinformatics Institute, Cambridge, UK. ${ }^{2}$ Wellcome Sanger Institute, Cambridge, UK.

${ }^{3}$ GlaxoSmithKline, Stevenage, UK. ${ }^{4}$ Faculty of Medicine, Joint Research Center for Computational Biomedicine Aachen, RWTH Aachen University, Aachen, Germany. ${ }^{5}$ Open Targets, Cambridge, UK. ${ }^{6}$ Present address: Faculty of Medicine, Institute for Computational Biomedicine, Bioquant, Heidelberg University, Heidelberg, Germany.

Received: 21 January 2018 Accepted: 31 July 2018

Published online: 13 August 2018

\section{References}

1. Mali P, Yang L, Esvelt KM, Aach J, Guell M, DiCarlo JE, et al. RNA-guided human genome engineering via Cas9. Science. 2013;339:823-6.

2. Cong L, Ran FA, Cox D, Lin S, Barretto R, Habib N, et al. Multiplex genome engineering using CRISPR/Cas systems. Science. 2013;339:819-23.

3. Koike-Yusa H, Li Y, Tan E-P, Velasco-Herrera MDC, Yusa K. Genome-wide recessive genetic screening in mammalian cells with a lentiviral CRISPRguide RNA library. Nat Biotechnol. 2014;32:267-73.

4. Shalem O, Sanjana NE, Hartenian E, Shi X, Scott DA, Mikkelson T, et al. Genome-scale CRISPR-Cas9 knockout screening in human cells. Science. 2014;343:84-7

5. Wang T, Wei JJ, Sabatini DM, Lander ES. Genetic screens in human cells using the CRISPR-Cas9 system. Science. 2014;343:80-4

6. Tzelepis K, Koike-Yusa H, De Braekeleer E, Li Y, Metzakopian E, Dovey OM, et al. A CRISPR Dropout Screen Identifies Genetic Vulnerabilities and Therapeutic Targets in Acute Myeloid Leukemia. Cell Rep. 2016;17:1193-205. 
7. Steinhart Z, Pavlovic Z, Chandrashekhar M, Hart T, Wang X, Zhang X, et al. Genome-wide CRISPR screens reveal a Wnt-FZD5 signaling circuit as a druggable vulnerability of RNF43-mutant pancreatic tumors. Nat Med. 2017;23:60-8.

8. Meyers RM, Bryan JG, McFarland JM, Weir BA, Sizemore AE, Xu H, et al. Computational correction of copy number effect improves specificity of CRISPR-Cas9 essentiality screens in cancer cells. Nat Genet [Internet]. 2017; 49(12):1779-1784 Available from: https://doi.org/10.1038/ng.3984

9. Wang T, Yu H, Hughes NW, Liu B, Kendirli A, Klein K, et al. Gene Essentiality Profiling Reveals Gene Networks and Synthetic Lethal Interactions with Oncogenic Ras. Cell. 2017;168:890-903.e15.

10. Aguirre AJ, Meyers RM, Weir BA, Vazquez F, Zhang C-Z, Ben-David U, et al. Genomic Copy Number Dictates a Gene-Independent Cell Response to CRISPR/Cas9 Targeting. Cancer Discov. 2016;6:914-29.

11. Munoz DM, Cassiani PJ, Li L, Billy E, Korn JM, Jones MD, et al. CRISPR Screens Provide a Comprehensive Assessment of Cancer Vulnerabilities but Generate False-Positive Hits for Highly Amplified Genomic Regions. Cancer Discov. 2016;6:900-13.

12. Wang T, Birsoy K, Hughes NW, Krupczak KM, Post Y, Wei J, et al. Identification and characterization of essential genes in the human genome. Science. 2015;350:1096-101.

13. Iorio F, Knijnenburg TA, Vis DJ, Bignell GR, Menden MP, Schubert M, et al. A Landscape of Pharmacogenomic Interactions in Cancer. Cell. 2016; 166(3):740-54

14. Garnett MJ, Edelman EJ, Heidorn SJ, Greenman CD, Dastur A, Lau KW, et al. Systematic identification of genomic markers of drug sensitivity in cancer cells. Nature. 2012;483:570-5.

15. Hart T, Chandrashekhar M, Aregger M, Steinhart Z, Brown KR, MacLeod G, et al. High-Resolution CRISPR Screens Reveal Fitness Genes and GenotypeSpecific Cancer Liabilities. Cell. 2015;163:1515-26.

16. Subramanian A, Tamayo P, Mootha VK, Mukherjee S, Ebert BL, Gillette MA, et al. Gene set enrichment analysis: a knowledge-based approach for interpreting genome-wide expression profiles. Proc Natl Acad Sci U S A. 2005; 102:15545.

17. Yoshihama M, Uechi T, Asakawa S, Kawasaki K, Kato S, Higa S, et al. The human ribosomal protein genes: sequencing and comparative analysis of 73 genes. Genome Res. 2002:12:379-90.

18. Venkatraman $\mathrm{ES}$, Olshen $\mathrm{AB}$. A faster circular binary segmentation algorithm for the analysis of array CGH data. Comput Appl Biosci. 2007;23:657-63.

19. Olshen $A B$, Venkatraman ES, Lucito $R$, Wigler M. Circular binary segmentation for the analysis of array-based DNA copy number data. Biostatistics. 2004:5:557-72.

20. Seshan VE, Olshen A. DNAcopy: DNA copy number data analysis. R package version. 2016;1. https://bioconductor.org/packages/release/bioc/html/ DNAcopy.html.

21. Hart T, Moffat J. BAGEL: a computational framework for identifying essential genes from pooled library screens. BMC Bioinformatics. 2016;17:164.

22. Li W, Xu H, Xiao T, Cong L, Love Ml, Zhang F, et al. MAGeCK enables robust identification of essential genes from genome-scale CRISPR/Cas9 knockout screens. Genome Biol. 2014;15:554.

23. Doench JG, Fusi N, Sullender M, Hegde M, Vaimberg EW, Donovan KF, et al. Optimized sgRNA design to maximize activity and minimize off-target effects of CRISPR-Cas9. Nat Biotechnol. 2016;34:184-91.

24. Ong SH, Li Y, Koike-Yusa H, Yusa K. Optimised metrics for CRISPR-KO screens with second-generation gRNA libraries. Sci Rep. 2017;7:7384

25. Mermel CH, Schumacher SE, Hill B, Meyerson ML, Beroukhim R, Getz G. GISTIC2.0 facilitates sensitive and confident localization of the targets of focal somatic copy-number alteration in human cancers. Genome Biol. 2011;12:R41.

26. Greenman CD, Bignell G, Butler A, Edkins S, Hinton J, Beare D, et al. PICNIC: an algorithm to predict absolute allelic copy number variation with microarray cancer data. Biostatistics. 2010;11:164-75.

27. Vogelstein B, Papadopoulos N, Velculescu VE, Zhou S, Diaz LA Jr, Kinzler KW. Cancer genome landscapes. Science. 2013;339:1546-58.

28. R Core Team. R: A Language and Environment for Statistical Computing [Internet]. Vienna, Austria: R Foundation for Statistical Computing; 2016. Available from: https://www.R-project.org/

29. Goncalves E, Behan FM, Louzada S, Arnol D., et al. Tandem duplications lead to loss of fitness effects in CRISPR-Cas9 data. bioRxiv [Internet]. biorxiv.org; 2018; Available from: https://www.biorxiv.org/content/early/ 2018/05/25/325076.abstract
30. Anders S, Huber W. Differential expression analysis for sequence count data. Genome Biol. 2010;11:R106.

31. Garcia-Alonso L, lorio F, Matchan A, Fonseca N, Jaaks P, Peat G, et al. Transcription Factor Activities Enhance Markers of Drug Sensitivity in Cancer. Cancer Res. 2018;78:769-80.

32. Barretina J, Caponigro G, Stransky N, Venkatesan K, Margolin AA, Kim S, et al. The Cancer Cell Line Encyclopedia enables predictive modelling of anticancer drug sensitivity. Nature. 2012;483:603-7.

33. Gao J, Aksoy BA, Dogrusoz U, Dresdner G, Gross B, Sumer SO, et al. Integrative analysis of complex cancer genomics and clinical profiles using the cBioPortal. Sci Signal. 2013;6:11.

34. Robin X, Turck N, Hainard A, Tiberti N, Lisacek F, Sanchez J-C, et al. pROC: an open-source package for $\mathrm{R}$ and $\mathrm{S}+$ to analyze and compare ROC curves. BMC Bioinformatics. 2011;12:77.

\section{Ready to submit your research? Choose BMC and benefit from:}

- fast, convenient online submission

- thorough peer review by experienced researchers in your field

- rapid publication on acceptance

- support for research data, including large and complex data types

- gold Open Access which fosters wider collaboration and increased citations

- maximum visibility for your research: over $100 \mathrm{M}$ website views per year

At BMC, research is always in progress.

Learn more biomedcentral.com/submissions 